\title{
Development of a Triplet-Triplet Absorption Ruler: DNA- and Chromatin-Mediated Drug Molecule Release from a Nanosurface
}

Sudeshna Das Chakraborty, ${ }^{\dagger}$ Abhishek Sau, ${ }^{\dagger}$ Denis V. Kuznetsov, ${ }^{\S}$ Amrita Banerjee, ${ }^{\ddagger}$ Munmun Bardhan, ${ }^{\dagger}$ Maireyee Bhattacharya, ${ }^{\dagger}$ Dipak Dasgupta, ${ }^{\ddagger}$ Samita Basu, ${ }^{\dagger *}$ Dulal Senapati ${ }^{+*}$

${ }^{\dagger}$ Chemical Sciences Division and ${ }^{\ddagger}$ Biophysics and Structural Genomics Division, Saha Institute of Nuclear Physics, 1/AF Bidhannagar, Kolkata 70oo64, India

${ }^{\S}$ Department of Functional Nanosystems and High Temperature Materials, National University of Science and Technology 'MISiS', Leninsky, Prospect 4, 119049 Moscow, Russia

Triplet-triplet absorption or laser flash photolysis, SERS, NSET, Drug, Sgr, ctDNA, chromatin 
Time resolved T-T absorption spectra (Figure S1) of Sgr $[100 \mu \mathrm{M}]$ show an overall decrease in optical density (OD) from 560 to $650 \mathrm{~nm}$ with a gradual increase in time, which indicates that all of these peaks are of the same origin. The origin of these three T-T absorption peaks is not as straightforward as it is observed for the S-S absorption spectra (UV-vis). Because of molecular state mixing and crossing between singlet and triplet states (intersystem crossing) to initiate a T$\mathrm{T}$ transition, it is difficult to define the actual nature of the originating or destination state without knowing the actual weightage of singlet-triplet state mixing, and we may need excitedstate molecular geometry optimization to infer their actual state identification, which we have not mentioned in this article.

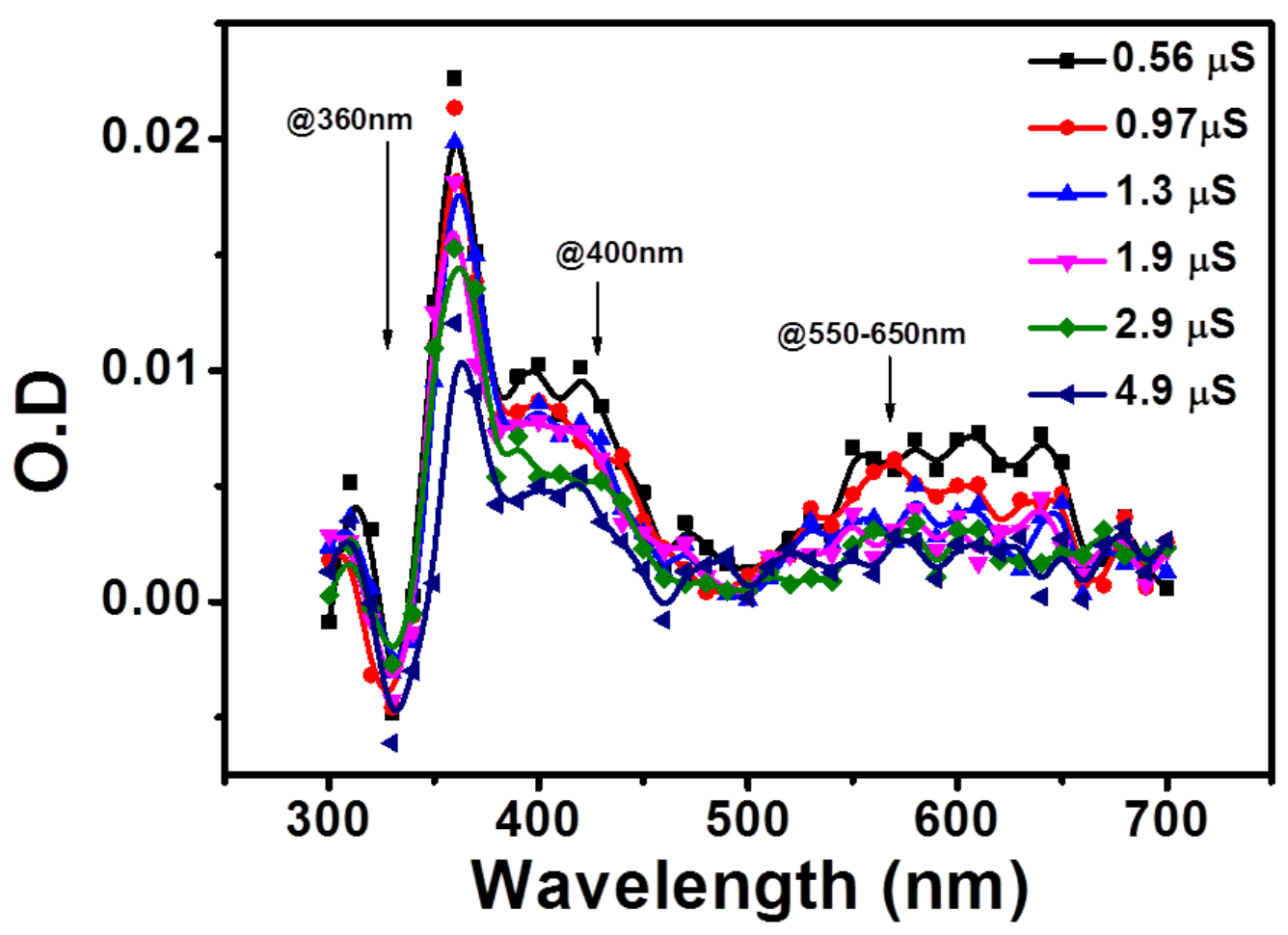

Figure S1. Time resolved T-T absorption spectra of Sgr $[100 \mu \mathrm{M}]$ show an overall decrease in optical density (OD) from 560 to $650 \mathrm{~nm}$ with a gradual increase in time, which indicates that all of these peaks are of the same origin. 
By considering GNP as an efficient fluorescence quencher, we analyzed fluorescence quenching of Sgr according to the Stern-Volmer equation

$\mathrm{F}_{0} / \mathrm{F}=1+\mathrm{K}_{\mathrm{SV}}[\mathrm{Q}]$

where, $\mathrm{F}_{0}$ and $\mathrm{F}$ are the fluorescence intensities of Sgr $(10 \mu \mathrm{M})$ in the absence and presence of GNPs, respectively, $\mathrm{K}_{\mathrm{SV}}$ is the Stern-Volmer constant, and [Q] is the concentration of the quencher, that is, AuNPs. The depicted Stern-Volmer plot (Figure S2) shows a deviation from linearity, which indirectly proves that the quenching route is a mixture of static and dynamic processes.

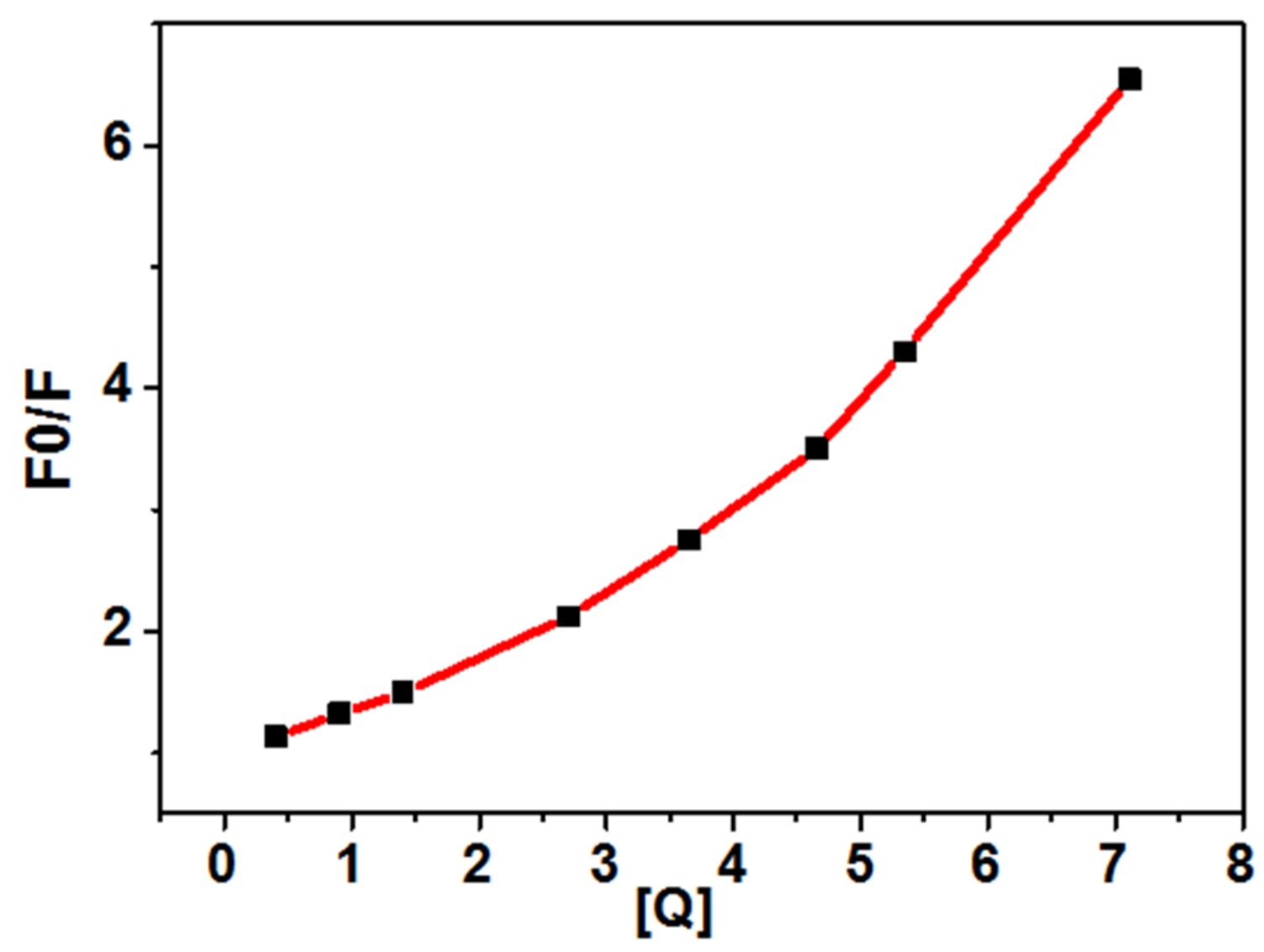

Figure S2. Variation of fluorescence quenching with the concentration of quencher (0.4-7.1nM). Here 20-25 nm TSC stabilized AuNPs acts as a quencher and Sgr as the fluorophore. 
After complexation of Sgr with citrate stabilized gold nanoparticles (20-25nm), the Au-Sgr complex is allowed to stand for $1 \mathrm{~h}$ as a control experiment to check whether the release of Sgr from AuNP surface is ctDNA/chromatin-mediated or it is released with time from the surface as a kinetically controlled process. From Figure S3 it is clear that there was no substantial release of Sgr in the absence of ctDNA and chromatin as biological scaffolds. This proves that the drug release is purely mediated by the presence of ctDNA and chromatin not as a kinetically controlled process.

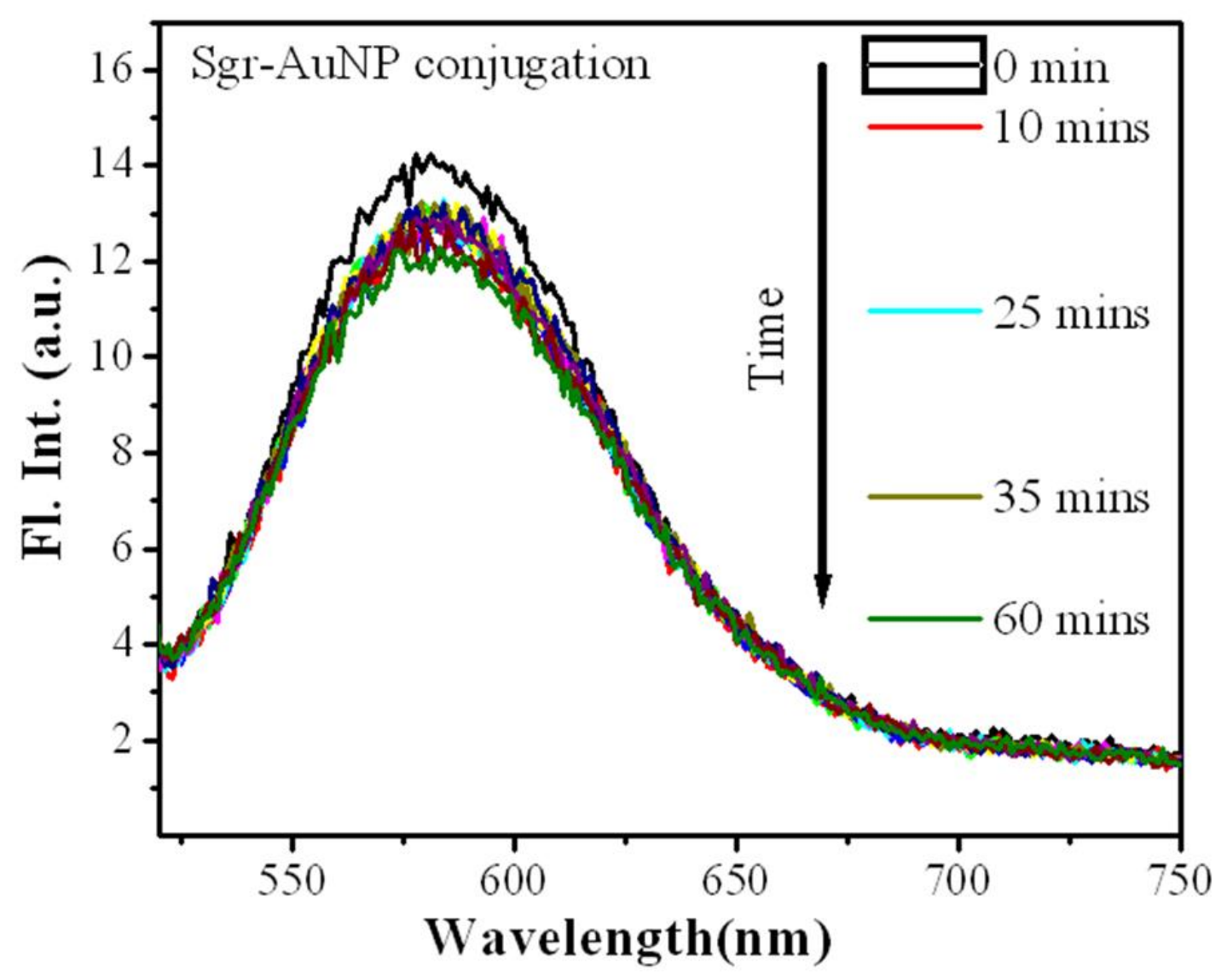

Figure S3. Variation of fluorescence intensity, obtained from Sgr-AuNP complex, with time without the addition of ctDNA and chromatin as active bio-scaffolds. 